\title{
ESTUDIO TÉCNICO ECONÓMICO COMPARADO DE LOS DESENGRASANTES DEL SECTOR METALMECÁNICO DE LA CIUDAD DE CALI
}

\section{COMPARATIVE ECONOMIC TECHNICAL STUDY OF DEGREASERS IN THE METALWORKING SECTOR OF THE CITY OF CALI}

\author{
Luis Miguel Bolaños Nazareno y Alejandro Cárdenas Rojas \\ Semillero de investigación Edward Deming \\ Universitaria Cooperativa de Colombia \\ Cómo citar este artículo: \\ Bolaños Nazareno, L.M. y Cárdenas Rojas, A. (2021). Estudio técnico económico comparado de los desengrasantes del sector metalme- \\ cánico de la ciudad de Cali. Revista Sapientía, 13 (26), 60-69.
}

\section{RESUMEN}

La presente investigación tiene como objetivo el estudio y análisis de tipo cuantitativo y cualitativo de los desengrasantes más utilizados en los principales talleres metalmecánicos de la ciudad de Santiago de Cali. Esto con el fin de conocer aspectos, tanto técnicos como económicos, para incursionar a futuro en el mercado con el emprendimiento de un producto desengrasante y cumplir con expectativas en relación costo-beneficio, fichas técnicas, fabricación del producto con agentes biodegradables amigables con el medio ambiente, procesos direccionados a una adecuada manipulación del producto y aplicabilidad oportuna a la ventaja del mismo. La investigación comprende dos fases de estudio, en la primera se describen una serie de estudios y análisis de fichas técnicas de productos desengrasantes del sector metalmecánico, de la información derivada del cuestionario y finalmente unos criterios de estudio técnico-económico comparando la variedad de productos desengrasantes referenciados y encuestados en el sector metalmecánico.

\section{PALABRAS CLAVE}

Desengrasante, industria metalmecánica, estudio técnico, estudio económico, biodegradable.

\section{ABSTRACT}

The present research aims to study and analyze the quantitative and qualitative type of degreasers most used in the main metalworking workshops in the city of Santiago de Cali. This in order to know aspects, both technical and economic, to enter the market in the future with the undertaking of a degreasing product and meet expectations in relation to cost-benefit, technical specifications, product manufacturing with environmentally friendly biodegradable agents. environment, processes aimed at an adequate handling of the product and timely applicability to its advantage. The research comprises two study phases, the first describes a series of studies and analysis of technical sheets of degreasing products in the metalworking sector, the information derived from the questionnaire and finally some technical-economic study criteria comparing the variety of degreasing products. referenced and surveyed in the metalworking sector.

\section{KEYWORDS}

Degreaser, industry metalworking, technical study, economic study, biodegradable. 


\section{INTRODUCCIÓN}

El sector metalmecánico abastece a las industrias manufactureras destinadas a la fabricación, reparación, ensamble y transformación del metal. De esta manera, interviene en la elaboración de una amplia gama de productos y servicios indispensables para el desarrollo de la economía, motivo por el cual se encuentra relacionada a través de insumos claves con otros sectores industriales como el automotriz, construcción, transporte, minería y agricultura (MTESS, 2016). Por lo cual, entre los residuos que pueden ser generados en los talleres metalmecánicos y que representan niveles altos de peligrosidad se encuentran aceites usados, virutas metálicas, elementos impregnados de sustancias químicas y tóxicas para la salud; también materias primas que pueden ser inflamables y corrosivas para su previa manipulación en los diferentes procesos productivos de esta industria como tal.

De acuerdo con lo planteado es necesario implementar estrategias que permitan la reducción y prevención integral de impactos y riesgos ambientales. Por ello, la implementación de herramientas de gestión ambiental, tales como una producción más limpia y amigable con el medio ambiente, se convierte en un factor determinante dentro de la estructura productiva y organizacional de las empresas o talleres metalmecánicos. Es así como la industria metalmecánica se ha ganado un importante espacio por su potencial para satisfacer la creciente demanda mundial de sus productos, esto ha impulsado a que un gran número de empresas sean certificadas con normas ISO, mediante la implementación de buenas prácticas que incluyen modificaciones en el proceso productivo para reducir la carga contaminante en el agua residual vertida a fuentes hídricas, cambios de materia prima que reduzcan generación de residuos contaminantes, entre otras (MTESS, 2016; Arias et al., 2007).

En el contexto nacional, la industria metalmecánica en el Valle del Cauca ha surgido últimamente con buenos datos de exportación de productos y demás respecto a otros departamentos del país. Por ejemplo, tuvo unas proyecciones de $15,7 \%$ en los primeros diez meses del año 2018 (Informe \# 109, enfoque competitivo, cámara comercio año 2018).

Alrededor de esta gama de productos, fabricaciones, equipos de operatividad y demás es de suma importancia la utilización de productos desengrasantes para asegurar la limpieza de esta serie de partes y piezas, ya que gracias a estos productos se garantiza la salubridad de muchos espacios, partes y elementos que necesitan a diario estar libres de partículas extrañas.

\section{MARCO TEÓRICO Antecedentes}

Así como el sector doméstico ha tenido por años este problema, las industrias desde sus inicios han presentado este inconveniente requiriendo mantener sus instalaciones y procesos en óptimas condiciones sanitarias. De ahí la necesidad de productos desengrasantes que garantizaran la eliminación de impurezas, cuyo uso es una práctica común desde hace décadas.

Al examinar bibliográficamente los antecedentes con relación a la fabricación de productos desengrasantes, se encuentra que uno de los primeros fue creado en 1953, en un pequeño laboratorio en San Diego, California, por la compañía Rocket Chemical Company, donde su equipo de tres personas se propusieron crear una línea de solventes y desengrasantes para la prevención de la oxidación para uso en la industria aeroespacial. Les tomó 40 intentos para que su fórmula de desplazamiento del agua funcionara, pero en el intento 40 lo hicieron bien a lo grande. WD-40 significa desplazamiento de agua, 40a fórmula. El nombre fue tomado directamente del libro de laboratorio utilizado por el químico que desarrolló el producto (WD-40 Company, 2021).

Por otro lado, en materia de innovación en el año 1949 el señor Jaume Lloreda funda una empresa de recubrimientos metálicos. Recubrir las soldaduras de joyería fue el primer paso para iniciarse plenamente en los recubrimientos metálicos de todo tipo, con el pasar del tiempo la empresa creció y ya ofrecía múltiples servicios, de tal manera que, a mediados de los 70, la necesidad interna de limpiar las piezas que se envían a los clientes es la causa de la aparición de los detergentes. En el intento de encontrar una solución a una necesidad interna, Jaume Lloreda cambió el rumbo de su empresa, decidió desarrollar sus propios productos. En 1977 se crea la fórmula para un producto desengrasante de uso interno llamado $\mathrm{KH}-7$ una fórmula que funcionaba muy bien contra la grasa.

\section{DEFINICIÓN Y FUNCIONAMIENTOS DE LOS DESENGRA- SANTES}

La Real Academia de la Lengua definió la palabra desengrasante como: "dicho de un producto que desengrasa (II quita la grasa)" (Real Academia Española, 2020). De acuerdo a diferentes fuentes, los desengrasantes se definen como sustancias químicas 
diseñadas para remover grasas, estos productos por su composición química son capaces de eliminar las impurezas más difíciles de arrancar lo que garantiza una limpieza total de las superficies.

Por otra parte, la corporación DALMAS Protección y Pinturas define desengrasante como aquella sustancia de naturaleza ácida, neutra o alcalina capaz de eliminar aceites y grasas de una superficie cualesquiera. Pueden ser formulados mediante sustancias naturales como de sustancias artificiales y su función es la de remover los aceites y grasas mediante una reacción química (DALMAR PROTECCIONES Y PINTURAS, 2015).

\section{TIPOS DE DESENGRASANTES}

En el mercado se puede encontrar variedad de desengrasantes con diferentes tipos y funciones, los cuales son utilizados en diferentes materiales, productos o máquinas, ya que sus componentes químicos actúan efectivamente.

Los componentes de los desengrasantes pueden variar, tanto los fabricados con productos naturales, que en muchos casos son muy amigables con el ambiente, como los que son a base de componentes artificiales, ambos cumplen el mismo objetivo: "eliminar la grasa". $\mathrm{Su}$ diferencia radia en el tipo de fórmula o componente químico que contenga el desengrasante y así mismo serán sus efectos en los diferentes campos de aplicación.

Dentro de esta clasificación están:

Desengrasante formulado base disolvente: los cuales son sido desarrollados para disolver los aceites y grasas de las superficies mediante una acción química, se utiliza especialmente para obtener como resultados superficies impecables.

Desengrasante formulado en base de agua: esta clase de desengrasante elimina los aceites y grasas que se encuentren sobre las superficies de cualquier material por medio de procesos de emulsión, es decir, que las moléculas de desengrasante inorgánico base agua actúen sobre las moléculas de aceite despegando esta impureza de las superficies.

Desengrasante industriales: esto son desengrasantes formulados para actuar sobre todo tipo de superficies y su función es erradicar suciedades que cuestan más trabajo eliminar como los son machas de aceite y grasas, por su composición química. Este producto tiene mayor capacidad de limpieza que cualquier otro tipo de desengrasante, por tal razón es utilizado exclusivamente en el sector industrial.

Desengrasantes biodegradables: es una mezcla de agentes emulsificantes, humectantes, surfactantes, solventes e inhibidores de corrosión, en base acuosa para obtener un desengrasante para la industria petrolera, minera e industria en general (DALMAR PROTECCIONES Y PINTURAS, 2015).

\section{TIPO Y POSICIONAMIENTO DE LOS DESENGRASANTES EN LA CIUDAD DE SANTIAGO DE CALI}

A continuación, se listan algunos desengrasantes usados en Santiago de Cali, de acuerdo con sus características, en tanto no se puede pasar por alto el impacto que estos productos pueden tener en el medioambiente.

\begin{tabular}{|c|c|c|}
\hline EMPRESA & CARACTERÍSTICA & PRODUCTOS \\
\hline \multirow[t]{4}{*}{$\begin{array}{l}\text { CLEAN } \\
\text { MASTER }\end{array}$} & \multirow{4}{*}{$\begin{array}{l}\text { Fabricantes de productos de } \\
\text { calidad para la limpieza } \\
\text { desinfección }\end{array}$} & $\begin{array}{l}\text { DETERGENTE LIQUIDO GOLD: } \\
\text { multiuso superconcentrado }\end{array}$ \\
\hline & & $\begin{array}{l}\text { DETERGENTE CLORADO: } \\
\text { desengrasante alcalino }\end{array}$ \\
\hline & & $\begin{array}{c}\text { DETERGENTE LIQUIDO PH ALCALINO: } \\
\text { desengrasante multiuso. }\end{array}$ \\
\hline & & $\begin{array}{l}\text { DETERGENTE 250: detergentes } \\
\text { desengrasantes de alta productividad, } \\
\text { se usa para trabajos muy pesados }\end{array}$ \\
\hline \multirow[t]{6}{*}{ VELQUÍMICOS } & \multirow{6}{*}{$\begin{array}{l}\text { Empresa dedicada a la } \\
\text { comercialización de } \\
\text { productos químicos para } \\
\text { diferentes sectores } \\
\text { industriales. }\end{array}$} & DESENGRASANTES: \\
\hline & & INDUSTRIALES \\
\hline & & Hidrosolubles \\
\hline & & Reutilizables \\
\hline & & No Inflamables. \\
\hline & & $\begin{array}{c}\text { PRODUCTOS DE ASEO GENERAL: } \\
\text { Hipoclorito de sodio, Ácido nítrico, } \\
\text { Ácido oxálico, Detersin, } \\
\text { Desengrasante, Ácido sulfónico y } \\
\text { Creolina. }\end{array}$ \\
\hline $\begin{array}{l}\text { DIMARQUIM } \\
\text { S.A.S. CALI }\end{array}$ & $\begin{array}{l}\text { Fabricantes y distribuidores } \\
\text { de desengrasante. También } \\
\text { comercializa productos } \\
\text { químicos para la industria. }\end{array}$ & $\begin{array}{c}\text { Desengrasante industrial Dimarquin: } \\
\text { es el mejor aliado para la limpieza, al } \\
\text { ser un producto sumamente potente } \\
\text { diseñado especialmente para eliminar } \\
\text { la suciedad más persistente y difícil de } \\
\text { suprimir. }\end{array}$ \\
\hline $\begin{array}{l}\text { QUÍMICOS } \\
\text { CALIMA }\end{array}$ & $\begin{array}{c}\text { Fabricante y comercializadora } \\
\text { de productos para el aseo, } \\
\text { perfumería fina y } \\
\text { multifuncional. }\end{array}$ & $\begin{array}{l}\text { Desengrasante Multiusos EL FUERTE. } \\
\text { Desengrasante Multiusos K-lima. }\end{array}$ \\
\hline \multirow{4}{*}{$\begin{array}{l}\text { DETERQUÍN \& } \\
\text { CÍA LTDA }\end{array}$} & \multirow{4}{*}{$\begin{array}{c}\text { Es una empresa colombiana, } \\
\text { fundada en 1988, dedicada a } \\
\text { la producción y distribución } \\
\text { de detergentes para la } \\
\text { industria y el sector hogar. }\end{array}$} & DESENGRASANTE F-30: \\
\hline & & $\begin{array}{l}\text { Detergente líquido de alto poder } \\
\text { desengrasante para limpieza manual y } \\
\text { automática de utensilios de cocina y } \\
\text { áreas engrasadas. }\end{array}$ \\
\hline & & DETERPON 90: \\
\hline & & $\begin{array}{l}\text { Detergente y desengrasante alcalino } \\
\text { para el lavado y mantenimiento de } \\
\text { superficies con residuos de aceites o } \\
\text { grasa carbonizada. }\end{array}$ \\
\hline
\end{tabular}

Tabla 1. Principales empresas distribuidoras de desengrasantes Fuente: elaboración propia 
En un mundo industrializado donde el comercio es extenso y la competencia es cada vez más difícil, los consumidores son más exigentes, por lo tanto, es necesario asegurarse de que el proyecto que vas a llevar a cabo llegue a cubrir las mejores expectativas. Por tal razón, se requiere que antes de iniciar con un proyecto se realice una evaluación detallada del mismo, para con ello tener información que conlleve a reducir los riesgos económicos y demás que pueden generar las decisiones erróneas.

\section{METODOLOGÍA}

Durante el desarrollo de la investigación, el equipo de trabajo realizó y aplicó un diseño metodológico basado en un modelo mixto, el cual ayudó a la previa caracterización combinada, enfocando en un primer plano la perspectiva cualitativa y en un segundo plano la perspectiva cuantitativa. Contemplando en un mismo estudio el análisis y el avance de los objetivos específicos del proyecto de investigación. Esta metodología de investigación prevé un estudio de mercado que permite dar profundidad y comprender mejor los procesos de enseñanza y aprendizaje, también proyectar diversas fuentes de información que se combinan de manera concreta para sustentar análisis más comprensivos acerca de la problemática planteada en el proyecto de investigación.

A partir de una metodología de investigación de mercado y considerando el objetivo general de la investigación: establecer las características técnico económicas que hacen viable la introducción de un nuevo producto desengrasante en los talleres de la industria metalmecánica de la ciudad de Santiago de Cali, los lineamientos metodológicos descritos van desde los estudios y criterios cuantitativos teóricos a los cualitativos prácticos para valorar y establecer los resultados. En este caso, la información cuantitativa (estudio evaluación económica) se pretende medir y cuantificar en términos de datos o números en los resultados previos que entregan los estudios preliminares.

En cuanto a la información cualitativa (estudio evaluación técnica, entorno o sector) analiza y proyecta la descripción completa y concisa de los resultados para facilitar una toma de decisión a corto, mediano y largo plazo para la ejecución del proyecto.
Por lo cual, para redefinir los resultados y discusión de esta investigación, se realizó un estudio bibliográfico identificando las características técnicas de los desengrasantes más utilizados en la industria metalmecánica con base en el estudio de las características de las fichas técnicas, también se desarrolló un trabajo de campo y virtual ejecutando encuestas en los diferentes talleres metalmecánicos del sector, para determinar los desengrasantes más utilizados en los principales talleres metalmecánicos de la ciudad de Santiago de Cali. Como resultado se obtuvo la descripción previa de diez (10) desengrasantes.

Por último, el estudio técnico económico comparado da como resultado la descripción de las mejores características a tener en cuenta para el desengrasante del emprendimiento. Así, el objetivo final de esta investigación consiste en iniciar la producción de un desengrasante que cumpla con las expectativas y necesidades del sector metalmecánico de la ciudad de Santiago de Cali.

\section{RESULTADO Y DISCUSIÓN}

En la inspección y temática para el estudio técnico económico se desarrolló una encuesta, seguido de un estudio técnico económico comparado. Por lo cual se referenció empresas y micro empresas de la industria metalmecánica de la ciudad de Santiago de Cali tomando como objeto de estudio empresas muy importantes en este segmento de mercado. De estas micro empresas y empresas conocemos, en primera instancia, que se desarrollan actividades a diario donde se utilizan desengrasantes para sus mantenimientos, reparaciones y demás, los cuales por sus propiedades de limpieza son de óptima necesidad en las actividades y procesos internos del gremio metalmecánico de la ciudad.

En la encuesta se encontraron datos cuantitativos y cualitativos, los cuales arrojan información muy importante para establecer un estudio técnico y económico comparado de los productos desengrasantes utilizados en la industria metalmecánica y así establecer las mejores características del desengrasante del emprendimiento. Por lo cual, a continuación, se realiza un análisis detallado de cada punto y descripción de la encuesta, 
tomando como referencia preguntas de tipo técnico y económico.

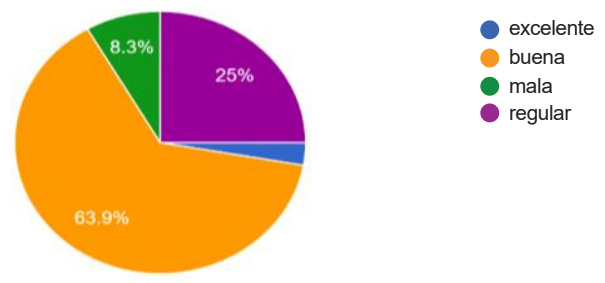

Fig. 1. ¿La información de tipo técnico y económico que ha recibido acerca de los desengrasantes es? Fuente: elaboración propia.

En la primera pregunta del cuestionario se visualiza un segmento de mercado de los desengrasantes, con criterios buenos para el desarrollo de un nuevo producto en el mercado, denotando un 63\%. Dado que la información general define un criterio de necesidad para la utilización de estos productos y más en el campo metalmecánico. De este modo, se tiene en cuenta la información de tipo técnico (fichas técnicas) e información económica (precios, oferta, demanda, presupuesto de compra de materia prima) para referenciar el desengrasante que se ajuste a la necesidad de compra y presupuesto del proveedor y cliente final.

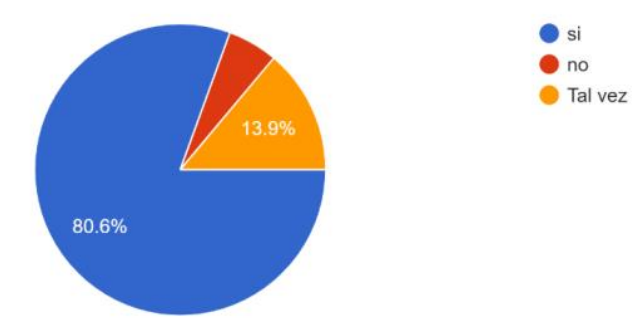

Fig. 2. ¿Actualmente compra algún producto desengrasante para ser utilizado en los servicios del taller? Fuente: elaboración propia.

En la Figura 2, que comprende una pregunta de tipo económico, puede observarse una importante demanda de los desengrasantes en este mercado metalmecánico. Este dato cuantitativo de un $80,6 \%$ no muestra la necesidad de oferta y demanda de los desengrasantes en la industria metalmecánica de la ciudad, porque son de total importancia en las actividades y labores de limpieza de partes en el taller de servicio. Es pertinente tener en cuenta que, por sus composiciones químicas, los desengrasantes tienen un papel destacado de ente de limpieza y calidad adecuada al terminar piezas de fabricación, moldes y demás en los talleres internos del sector metalmecánico.

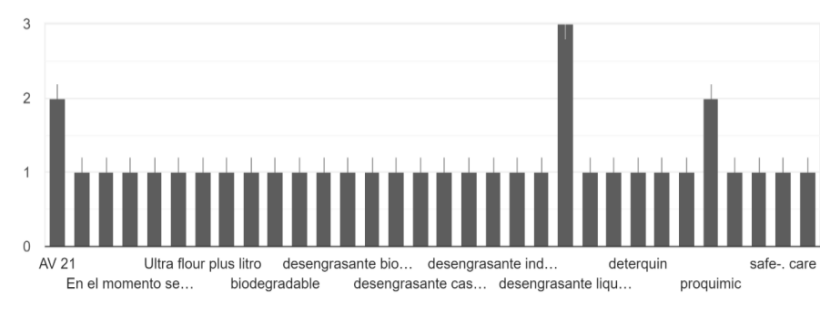

Fig. 3. ¿Qué tipo o marca de producto desengrasante utiliza en los servicios del taller? Fuente: elaboración propia.

Como ilustra la Figura 3, la pregunta define aspectos de tipo técnico. En este caso, muestra las características de tipo técnico que los productos desengrasantes biodegradables y desengrasantes de tipo industrial tienen en el mercado o industria metalmecánica de la ciudad. Estos criterios son de suma relevancia, dado que si se proyecta el desengrasante del emprendimiento con composición química y agentes biodegradables se obtendrá una demanda aceptable en el mercado y buena relación comercial con nuevos clientes al momento de vender este producto amigable con el medio ambiente.
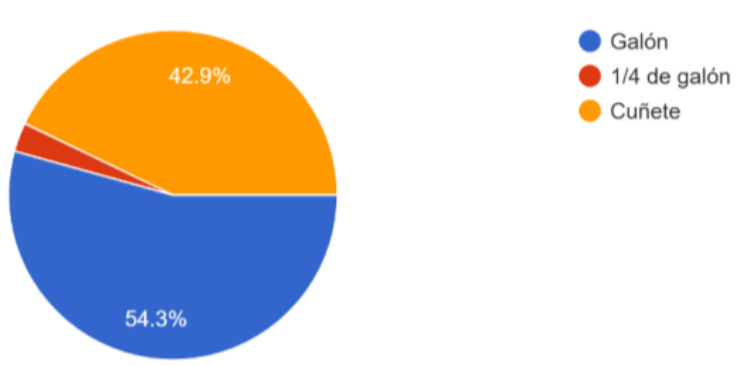
Fig. 4. ¿En qué presentación comercial compra el producto desengrasante? Fuente: elaboración propia.

La Figura 4 ilustra el porcentaje de respuesta a la pregunta: ¿En qué presentación comercial compra el producto desengrasante? De lo cual se infiere que un $54,3 \%$ de la industria metalmecánica de la ciudad de Santiago de Cali, prefiere la presentación y distribución en galón. Esto permite proyectar un empacado y venta del producto en esta presentación, que tendrá una buena aceptación por parte de los futuros clientes. 


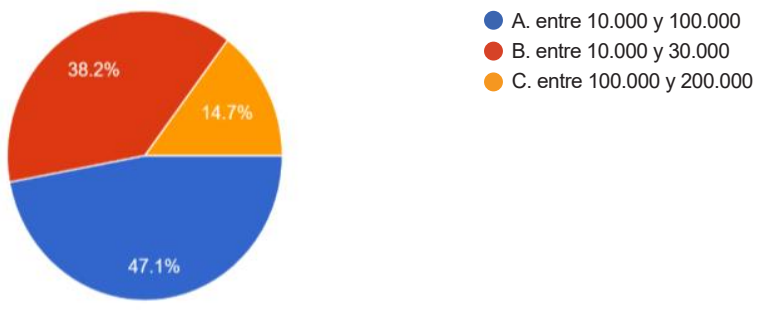

Fig. 5. ¿Cuál es el rango de precio de compra de adquisición del producto desengrasante en galones? Fuente: elaboración propia.

Como se muestra en la Figura 5, en este tipo de pregunta podemos evaluar aspectos económicos. La pregunta muestra el costo o rango de precios de los productos desengrasantes de la industria metalmecánica, específicamente en presentación de galón. El 47,1 \% indica el costo de venta del desengrasante como visión de emprendimiento, lo cual es de suma importancia para establecer costos de la materia prima, presentación, distribución, almacenamiento, mano de obra y demás y así definir un precio justo en el rango aceptable y actual del mercado metalmecánico.

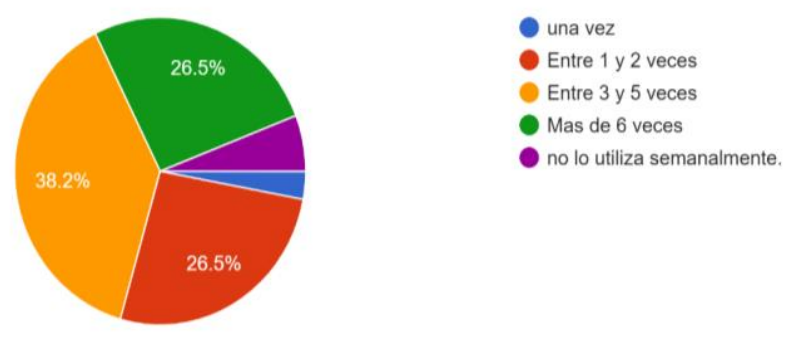

Fig. 6. ¿Con que frecuencia utiliza el producto desengrasante semanalmente? Fuente: elaboración propia.

En la Figura 6 se muestran aspectos tipo técnico económico consultados en la encuesta. Al respecto, con una frecuencia de uso mayor en los principales talleres metalmecánicos del sector se obtendrá una mayor demanda en el mercado al momento de realizar distribuciones. En cuanto al factor económico, no define proyección de ventas semanal y mensual con costos de mayor ingreso económico por aumento de ventas.

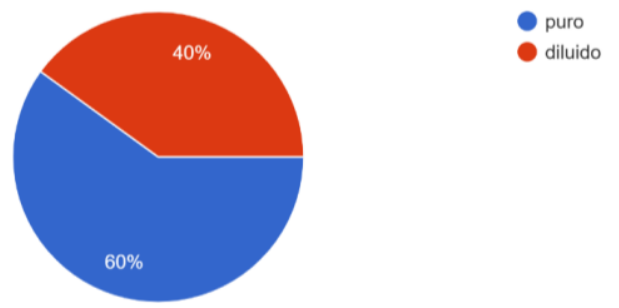

Fig. 7. ¿Como utiliza el tipo de desengrasante? Fuente: Elaboración Propia

La Figura 7 refiere aspectos técnicos consultados. El informe del cuestionario muestra que un $60 \%$ utiliza los desengranes de tipo puro y en ocasiones diluido con agua. Esto con el fin de tener una mayor y adecuada aplicación de los agentes químicos de limpieza del desengrasante en los principales talleres metalmecánicos de la industria, en procesos de limpieza de partes mecánicas y lavado de equipos internos del taller después de labores de operación.

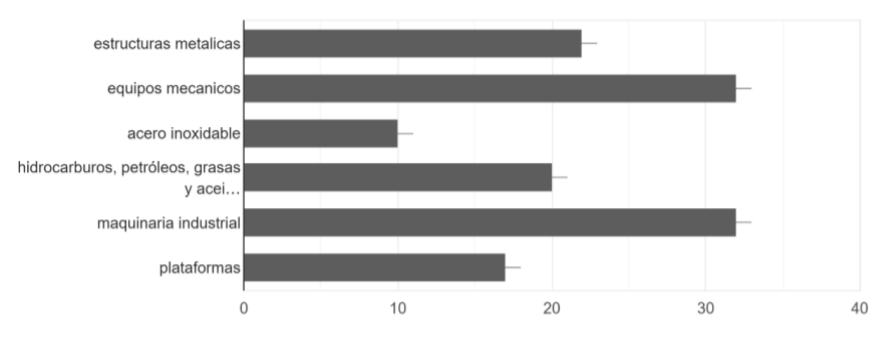

Fig. 8. ¿Para qué tipo de limpieza o servicio utiliza más los productos desengrasantes en el taller? Fuente: Elaboración Propia

La pregunta, cuyos resultados presenta la Figura 8, describe aspectos técnicos, de aplicación o enfoque del producto desengrasante del emprendimiento, los cuales permitirán direccionar los agentes químicos de fabricación, ficha técnica y demás en aplicabilidad para limpieza de maquinaria industrial y equipos mecánicos; estos enfoques evidenciados en el mercado proyectan a tener una mejor visual y poder segmentar en criterios técnicos el desengrasante de emprendimiento, dado que la maquinaria industrial juega un papel importante en el desarrollo de muchas empresas del sector de la ciudad de Cali y sus alrededores. Por otro lado, los equipos mecánicos son de uso diario en procesos internos de las plantas de producción y talleres metalmecánicos a nivel general. 


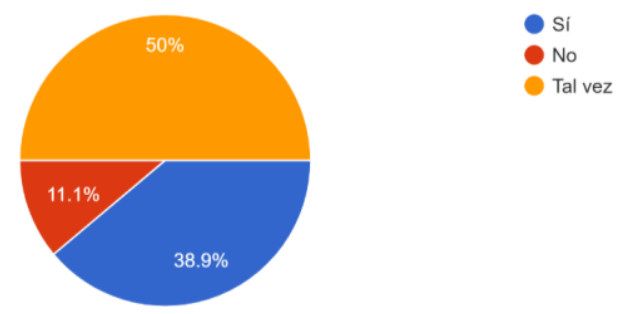

Fig. 9. ¿probaría otro producto desengrasante distinto al de uso actual? Fuente: Elaboración Propia

Los resultados que ilustra la Figura 9 con relación a una pregunta de tipo económico-comercial, arroja datos e información para proyectar una buena aceptación para un nuevo producto desengrasante que se proyecta introducir en el mercado. Con la aceptación parcial de los talleres metalmecánicos encuestados se podrían realizar pruebas del producto desengrasante de emprendimiento, con el fin de ganar participación e importancia en este tipo de mercado.

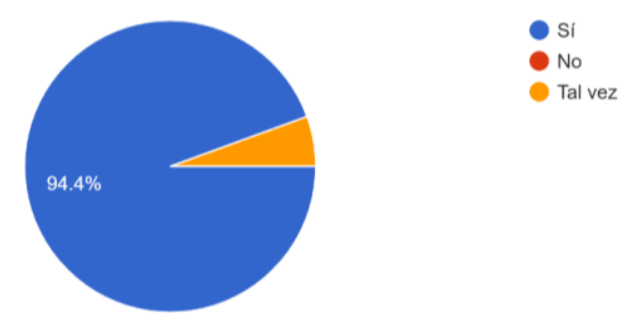

Fig. 10. ¿Está de acuerdo en la inversión y utilización de productos desengrasantes con agentes biodegradables? Fuente: Elaboración Propia

La Figura 10 presenta los resultados de la pregunta ¿Está de acuerdo en la inversión y utilización de productos desengrasantes con agentes biodegradables?, la cual da un parámetro importante en temas técnicos y económicos a tener en cuenta para la nueva incursión del producto desengrasante del emprendimiento. Los resultados de la encuesta muestran una aceptación de un $94,4 \%$ respecto a la utilización de nuevos productos desengrasantes con agentes biodegradables y amigables con el medio ambiente. Este segmento de productos biodegradables en el mercado metalmecánico de los principales talleres de la ciudad ha tenido buena acogida, dando muestra de la importancia de utilizar agentes químicos amigables según normas internacionales y nacionales que ayudan a controlar afectaciones directas del medio ambiente. En este sentido, es importante incursionar con el producto desengrasante de emprendimiento en este plus de mercado para tener una aceptación mejor en comparación con otros desengrasantes del mercado sin esta propiedad físico-química.

Teniendo en cuenta los datos arrojados por la encuesta realizada en los principales talleres metalmecánicos de la ciudad de Santiago de Cali, se evidenció que estos utilizan diferentes tipos y marcas de desengrasantes. $\mathrm{Al}$ hacer una búsqueda detallada de cada referencia, algunas no se encontraron en los buscadores, lo cual nos lleva a determinar que en algunos casos se trata de productos informales $y / 0$ fabricados por microempresas informales o sin registro en cámara de comercio. Por esta razón, como criterio de análisis para el estudio técnico económico comparado se tuvo en cuenta 10 desengrasantes para tomar referencia detallada de los mismos y así establecer las mejores características del desengrasante de emprendimiento. Es por eso que, a continuación, se describen las mejores características del análisis del estudio técnico económico comparado. En el segmento técnico comparado se evidenció un desarrollo de análisis tipo cualitativo sobre la información actual de las encuestas y marcos referenciales de páginas web y base de datos de los 10 desengrasantes, tomando como objeto de estudio las fichas técnicas y demás. Por lo anterior, y como conclusión del estudio comparado, se describe lo siguiente:

- Se requiere incursionar en el mercado con un producto desengrasante de emprendimiento con agentes de limpieza biodegradables.

- Tener en cuenta registros y certificaciones nacionales e internacionales para soportes de calidad del producto desengrasante de emprendimiento.

- Fabricar el desengrasante de emprendimiento libre de agentes con fosfatos y sulfatos, para no generar daños en la calidad del agua al momento de realizar las diluciones correspondientes. 
- Nuestro desengrasante de emprendimiento debe ser fabricado con agentes de limpieza apropiados para reaccionar sobre cualquier superficie (superficies pintadas, oxidadas, acero inoxidable, etc.).

- Fabricar el desengrasante del emprendimiento con agentes de solución ácida compuesta por surfactantes, estabilizantes, dado que nos permite una mayor influencia en la zona de contacto entre el agua+aceite-grasa+desengrasante.

- Implementar el desengrasante con criterios de no inflamabilidad y sin sustancias abrasivas.

- El desengrasante tiene que ser $100 \%$ soluble en agua.

- El desengrasante debe tener un punto de ebullición de $100 \mathrm{C}^{\circ}$.

- Definir estabilidad y almacenamiento en condiciones normales con periodicidad de un año aproximado.

- El desengrasante debe de tener variedad de presentaciones para su distribución y comercialización, en las cuales, según informe de estudio comparado, se debe de incursionar con presentación por galón y cuñete.

- Dentro de las fichas técnicas del desengrasante de emprendimiento se debe de informar previamente un adecuado protocolo de almacenamiento y uso, en los cuales definir su rango de temperatura para almacenamiento. Por lo cual se debe proteger de temperaturas mayores de $+50^{\circ} \mathrm{C}$ y de la luz directa del sol. Un modo acorde de aplicación ya sea por inmersión, pistola o con brocha.

- El desengrasante de emprendimiento debe de estar fabricado con agentes de limpieza que no produzcan corrosión a las piezas al momento de lavarlas y que no dejen residuos de agentes de alta reacción química para descomponer y limpiar superficies con aceite, grasa, hidrocarburos y demás.

Para finalizar, en el segmento económico comparado se evidenció un análisis tipo cuantitativo sobre la información actual de las encuestas y marcos referenciales de páginas web y base de datos de los 10 desengrasantes, tomando como objeto de estudio las fichas técnicas y demás. Por lo anterior, se describe lo siguiente:

El equipo de trabajo, basándose en la encuesta realizada y en la tabla en la que se hace la comparación, en cuanto a la presentación más adecuada, se evidencia que la mayoría de las personas encuestadas compran sus productos desengrasantes en presentación de cuñete (5 galones). Teniendo en cuenta que el estudio se realizó a talleres formales e informales, se deduce que las necesidades no son las mismas en su totalidad, por lo tanto, se sugiere que la presentación más rentable es por galones. Al hacer una búsqueda de los precios presentación por galón, algunos productos son costosos como el desengrasante de motor Spangel, cuyo precio en el mercado por galón está en \$ 67.494; el desengrasante Green Power se muestra ventajoso ya que, aunque el cuñete cueste \$200.000, al hacer la división se denota que el galón costaría $\$ 40.000$, lo que también sucede con otros desengrasantes según la tabla realizada. Siguiendo la misma línea costo-beneficio en base a la tabla de relación de las características económicas de los desengrasantes se puede ver que el uso del producto Green Power es muy frecuente ( 3 a 5 veces a la semana), en comparación con el producto Wurth, Spangel y Acid Clean, los cuales tienen una frecuencia de uso igual o mayor. Aunque sus características técnicas son muy buenas, tienen una gran desventaja siendo más costosos que el desengrasante Green Power con una diferencia de precios entre \$27.000 y \$ 29.344 pesos por galón.

El desengrasante Weicon, aunque tiene un precio moderado, se puede apreciar que tiene una frecuencia de uso muy baja en comparación con todos los desengrasantes en mención (de 1 a 2 veces por semana), su canal de suministro es recomendado según la encuesta y se usa para las labores básicas de la metalmecánica: estructuras y maquinaria industrial. 
Aunque el estudio muestra que todos tienen algo en común en cuanto a su uso o aplicación, se destaca como el desengrasante más costoso el producto Acid Clean PL con un valor de \$ 69.344 por galón, utilizado con frecuencia $y$ no cuenta con agentes biodegradables, lo cual según la encuesta es una característica que a todos los encuestados les gustaría que tuvieran los desengrasantes que utilizan en los talleres metalmecánicos de la ciudad. Como dato importante para el grupo de trabajo, en materia de cuidado ambiental se denota que si el producto cuenta con agentes biodegradables aporta un valor agregado para los consumidores (talleres metalmecánicos) y puede justificar el costo del mismo, aunque en su mayoría los que cuentan con estas características tienen precios económicos tales como: Green plus, AV21 TR, DTR20 y Green Power.

\section{CONCLUSIONES}

Respecto a las revisiones bibliográficas, en los documentos hallados se referencia información relevante frente a puntos de vista o diferentes definiciones de los desengrasantes industriales. Igual sucede con los campos de aplicación que tienen los desengrasantes y con qué características deben contar para realizar un correcto proceso de limpieza de grasa, aceites y demás. Se concluye también que los desengrasantes se pueden clasificar en grupos, que en el mercado se pueden encontrar gran variedad de características según la efectividad y las necesidades de utilización, teniendo en cuenta que dentro de estos productos existen algunos que pueden ser muy buenos en la eliminación de grasas, pero con características que pueden ser muy dañinas para el ambiente y la integridad de las personas. Como un aporte al cambio, para algunos investigadores son importantes los productos con agentes que contengan características no tóxicas o dañinas al entorno, lo que evidencia que se pueden producir desengrasantes a base de recursos naturales, conservando su alto poder desengrasante industrial.

Para referenciar un producto desengrasante viable de acuerdo con el estudio realizado es importante considerar directrices a nivel muy específico, ya que el producto o futura idea de emprendimiento para incursionar en la industria de los desengrasantes debe tener criterios específicos, innovadores y concretos, puesto que actualmente la industria de los desengrasantes tiene un mercado muy competitivo, por la alta gama de productos desengrasantes en el mercado. Por lo cual, a continuación, se indican los futuros criterios tentativos para cumplir las necesidades actuales del mercado.

- Tener como base una excelente sustancia química limpiadora y fuertemente activa, la cual pueda remover efectivamente la grasa y todo tipo de mugre depositada en motores, maquinaria y equipo industrial del sector.

- Especificar el producto con una gravedad de $25^{\circ} \mathrm{C}$, un olor característico de disolvente, almacenamiento adecuado para determinar un rango de temperatura óptimo de guardado y un límite de almacenamiento máximo sugerido: 1 año.

- Incursionar en el mercado con un producto netamente biodegradable, ya que algunos productos relacionados anteriormente no toman como base la palabra biodegradable para fortalecer y minimizar a largo plazo las sustancias contaminantes.

A su vez, con base en la información analizada de la encuesta realizada a diferentes talleres en la ciudad de Santiago de Cali, se pudo deducir que estos consumidores tienen un promedio de aceptación bueno en cuanto a características técnicas de los desengrasantes que se comercializan en el mercado local. Sin embargo, como dato a favor para el grupo de trabajo actual en este proyecto, en materia de economía se pudo redefinir que la mayoría consumen o compran el producto en presentación elevada para su consumo promedio, lo cual, como conclusión del grupo de 
trabajo, se proyecta que el desengrasante de emprendimiento sea producido y fabricado en presentaciones adecuadas a la proporción de trabajo de estos diferentes talleres metalmecánicos de la ciudad.

- Definir criterios económicos, dado que en el mercado se encuentran diferentes precios de los productos desengrasantes según su presentación. Con esto se toma como referencia la presentación con mayor distribución y comercialización para competir con precios acordes a la demanda.

\section{REFERENCIAS BIBLIOGRÁFICAS}

Arias, G., Fernández, A., Hernández, L., Navarro, J., Orrego, A., Tobón, C., Vélez, A., Vinasco, L. (2007). Producción Más Limpia y Buenas Prácticas Ambientales en las MIPYME del Municipio de Itagüí. Recuperado de: http://unicesar.ambientalex.info/infoCT/Pmlit aguibuen.pdf

Acero, R. (2004). Oportunidades de producción más limpia en el sector de metalmecánica. Guía para empresarios. DIAGRAMACIÖN Comunidad i.

Dalmar Protecciones y Pinturas. (25 de febrero de 2015). ¿Qué es un desengrasante? http://blog. proteccionesypinturas.com/desengrasante-ind ustrial-que-es-un-desengrasante-guia-del-dese ngrasante-industrial/

Dermo. (2016). Importancia de los desengrasantes. https://dermo.com/importancia-de-los-desen grasantes/

MTESS. (2016). Ministerio de Trabajo, empleo y seguridad social. Manual de Buenas prácticas Industria Metalmecánica. Argentina.

ISO 25000. (2021). Evaluación de productos. https://iso 25000.com/index.php/evaluacion- productos

KH-7. (2021). Nuestra historia. https://kh7.es/ corporativo/historia/
Real Academia de la Lengua Española. (2020). Desengrasante. https://dle.rae.es/desengrasante ? $\mathrm{m}=$ form

SUMATEC. (5 de febrero de 2019). Desengrasantes industriales para la limpieza y mantenimiento de superficies. https://sumatec.co/desengrasantes-industriales-para-la-limpieza-y-mantenimi ento-de-su-superficies/

WD-40 Company. (2021). Our history. https:// wd40company.com/our-company/our-history/

\section{AUTORES}

Luis Miguel Bolaños Nazareno: estudiante de Ingeniería Industrial de la Universidad Cooperativa de Colombia, sede Santiago de Cali. Correo: luis.bolanosn@campusucc.edu.co

Alejandro Cárdenas Rojas: estudiante de Ingeniería Industrial de la Universidad Cooperativa de Colombia, sede Santiago de Cali. Correo: alejandro.cardenasr@campusucc.edu.co 